Chi $r$ ping and Sudden Excit at $i$ on of Ener get i c- Part i cl e- Dri ven Geodesi c Acoust i c Nodes in a Large Hel i cal Devi ce Experi ment

\begin{tabular}{|l|l|}
\hline $\begin{array}{l}\text { jour nal or } \\
\text { publ i cat i on ti t l e }\end{array}$ & Physi cal Revi ew Let ter s \\
\hline vol ume & 120 \\
\hline page r ange & 175001 \\
\hline year & 2018 04 27 \\
\hline URL & ht t p: //hdl . handl e. net /10655/00012650 \\
\hline
\end{tabular}




\title{
Chirping and sudden excitation of energetic-particle driven geodesic acoustic modes in a Large Helical Device experiment
}

\author{
Hao Wang (王 影), ${ }^{1, *}$ Yasushi Todo (藤堂 泰), ${ }^{1,2}$ Takeshi Ido (井戸 毅), ${ }^{1}$ and Yasuhiro Suzuki (鈴木 康浩) $)^{1,2}$ \\ ${ }^{1}$ National Institute for Fusion Science, National Institutes of Natural Sciences, Toki 509-5292, Japan \\ ${ }^{2}$ SOKENDAI (The Graduate University for Advanced Studies), Toki 509-5292, Japan
}

\begin{abstract}
Energetic-particle driven geodesic acoustic modes (EGAMs) observed in a Large Helical Device (LHD) experiment are investigated using a hybrid simulation code for energetic particles interacting with a magnetohydrodynamic (MHD) fluid. The frequency chirping of the primary mode and the sudden excitation of the half-frequency secondary mode are reproduced for the first time with the hybrid simulation using the realistic physical condition and the 3-dimensional equilibrium. Both EGAMs have global spatial profiles, which are consistent with the experimental measurements. For the secondary mode, the bulk pressure perturbation and the energetic particle pressure perturbation cancel each other out, and thus the frequency is lower than the primary mode. It is found that the excitation of the secondary mode does not depend on the nonlinear MHD coupling. The secondary mode is excited by energetic particles that satisfy both the linear and nonlinear resonance conditions, respectively, for the primary and the secondary modes.
\end{abstract}

PACS numbers: $52.35 . \mathrm{Fp}, 52.55 . \mathrm{Hc}, 52.65 . \mathrm{Ww}$

Geodesic acoustic mode (GAM) is an oscillatory zonal flow coupled with density and pressure perturbations in toroidal plasmas[1-4]. In the last decade, energeticparticle driven GAMs (EGAMs) were observed in Joint European Torus (JET)[5, 6], DIII-D[7-9], Large Helical Device (LHD)[10-14], and ASDEX-Upgrade[15]. In the DIII-D experiment, drops in neutron emission followed the EGAM bursts, suggesting beam ion losses[7]. Also, in the LHD experiment, anomalous bulk ion heating during the EGAM activity suggests a GAM channeling[12]. In addition, EGAM can interact with turbulence and affect the plasma confinement[16, 17]. The understanding of the EGAM thus is important for magnetic confinement fusion where the energetic particles need to be well confined and the bulk plasma needs to be efficiently heated. The EGAM has been studied extensively. It was demonstrated that the poloidal mode number of the EGAM is $m=0$ for potential and $m=1$ for density. Also, the EGAM is a global mode with the spatially uniform oscillation frequency. In addition, the EGAM frequency can be lower or higher than the conventional GAM frequency under different conditions[18-21]. The above discussion has been advanced theoretically, computationally, and experimentally.

Recently, in LHD an abrupt excitation of a halffrequency secondary mode was observed when the frequency of a chirping primary EGAM reached twice the GAM frequency[14]. The secondary mode is important because of its low frequency. The lower frequency mode has a lower phase velocity, thus, this mode interacts more easily with the thermal ions and transfers energy to ions. As a result, the plasma heating thus becomes easier. Since the appearance of the secondary mode is related to the neutral beam injection (NBI), the secondary mode may create an energy channel between the energetic particles and the bulk plasmas. A 1-dimensional simulation with the kinetic energetic particles and a nonlinear coupling coefficient between the primary and the secondary modes was used to reproduce these two modes[22]. The authors of Ref. [22] have claimed that the secondary mode is driven by the cooperative combination of fluid nonlinearity and kinetic nonlinearity.

MEGA[23-25], a hybrid simulation code for energetic particles interacting with a magnetohydrodynamic (MHD) fluid, is used for the simulation of EGAMs. In the MEGA code, the bulk plasma is described by the nonlinear MHD equations. The drift kinetic description and the $\delta f$ particle method are applied to the energetic particles.

A realistic 3-dimensional equilibrium generated by HINT code[26] is used for the simulation. This equilibrium data is based on the LHD shot \#109031 at time $t=4.94 \mathrm{~s}$. At that time, $t=4.94 \mathrm{~s}$, the EGAM activity is very strong, thus it is appropriate to reproduce the EGAM phenomenon in a simulation.

In the experiments of LHD, the EGAMs were observed under the bump-on-tail energetic particle distribution[12, 14]. Thus, in the present work, we implement the simulation with the same type of distribution. For the bumpon-tail distribution, the charge exchange is considered, and the velocity distribution is:

$$
f(v)=C\left(v^{3}+v_{c}^{3}\right)^{\frac{1}{3} \tau_{s} / \tau_{c x}-1},
$$

which is the same as the Eq.(1) of Ref.[21]. The $C$ is an integration constant, $v_{c}$ is the critical velocity, $\tau_{s}$ is slowing down time, and $\tau_{c x}$ is charge exchange time. The shape of the distribution function is controlled by the ratio of $\tau_{s} / \tau_{c x}$. For $\tau_{c x} \rightarrow \infty$, the $\tau$ ratio is 0 and the distribution function is the typical slowing-down type. With the increase of $\tau_{s} / \tau_{c x}$, the slowing down becomes insufficient gradually, and more energetic particles distribute in the high-energy region and form a bump-on-tail distribu- 
tion. The distribution function of $f(v)$ in the present letter is the same as the experimental observations[13, 14].

In addition, a Gaussian-type pitch angle distribution $g(\Lambda)$ is assumed for the energetic ions:

$$
g(\Lambda)=\exp \left[-\left(\Lambda-\Lambda_{\text {peak }}\right)^{2} / \Delta \Lambda^{2}\right],
$$

where $\Lambda_{\text {peak }}$ represents the pitch angle for the distribution peak and $\Delta \Lambda$ is a parameter to control the distribution width.

The parameters for the EGAM simulation are based on an LHD experiment[14]. Those parameters are $B_{0}=$ $1.5 \mathrm{~T}$, electron density $n_{e}=0.1 \times 10^{19} \mathrm{~m}^{-3}$, electron temperature at the magnetic axis $T_{e}=4 \mathrm{keV}$, and bulk plasma beta value on the magnetic axis equals to $7.2 \times 10^{-4}$. The counter-injected neutral beam energy is $E_{N B I}=175 \mathrm{keV}$. The safety factor $q$ profile is negative normal shear with the value 2.82 at the magnetic axis and 0.84 at the plasma edge. The major radius of the magnetic axis is $R_{0}=3.7 \mathrm{~m}$. Cylindrical coordinates $(R, \phi, z)$ are employed. For LHD equilibrium, there are 10 pitches in the toroidal direction. Since the toroidal mode number of GAM is $n=0$, for simplicity, only 1 pitch from $\phi=0$ to $\phi=0.2 \pi$ is used for the present simulation, while other pitches from $\phi=0.2 \pi$ to $\phi=2 \pi$ are obtained by periodic extension. This simplification is made to save computational resources and time. The numbers of grid points of this pitch in $(R, \phi, z)$ directions are $(128,64,128)$, respectively.

Both the chirping primary mode and the halffrequency secondary mode are reproduced by the MEGA code, as shown in Fig. 1. Figure 1(a) shows the poloidal velocity $v_{\theta}$ frequency spectrum including all the frequency components, and Fig. 1(b) shows $v_{\theta}$ evolution including only $50 \mathrm{kHz}$ and $100 \mathrm{kHz}$ components. The primary mode frequency chirps up in the nonlinear phase from $70 \mathrm{kHz}$ in the linear growth phase. The mode is saturated at $t=0.07 \mathrm{~ms}$, and then moves into the nonlinear phase. At $t=1.7 \mathrm{~ms}$, the frequency of the primary mode reaches to $102 \mathrm{kHz}$, and a secondary mode with frequency $f=51 \mathrm{kHz}$ is excited. The amplitudes of the primary mode and the secondary mode are close to each other. The simulated phenomenon is very similar to the experimental observation, as shown in Fig. 2 of Ref. [14]. This is the first simulation that reproduces both the primary mode and the secondary mode with a 3 -dimensional model and realistic input parameters.

The frequency of the primary mode is two times that of the secondary mode, and this frequency relation can be easily confirmed by Lissajous curves. Figure 2 shows the Lissajous curves between $d B_{\theta} / d t$ associated with the primary and the secondary modes. Panel (a) is plotted in the growth phase and panel (b) is plotted in the decay phase of the secondary mode, respectively. The phase locking is clearly shown in the figure, and indicates a coupling between the primary mode and the secondary mode. The Lissajous curves in the present simulation
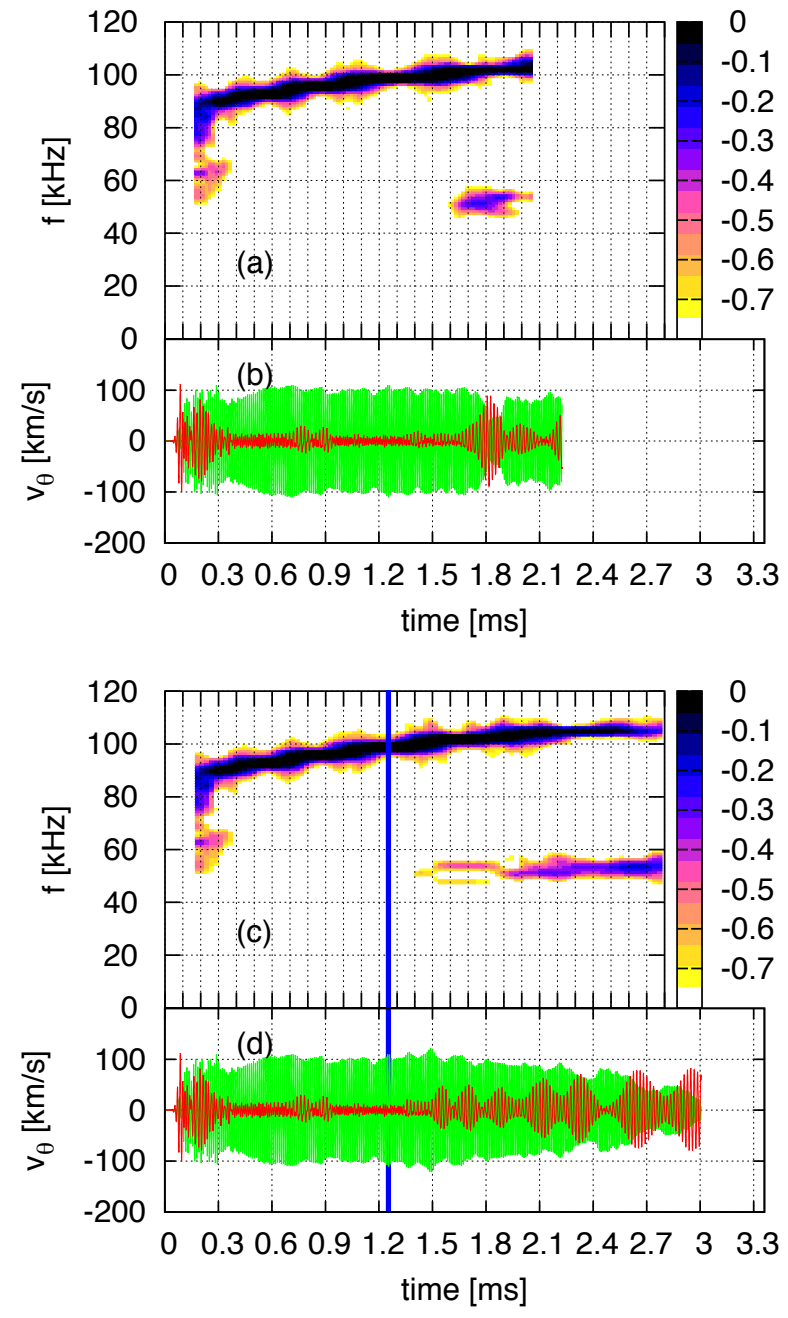

FIG. 1. The EGAMs in LHD are reproduced by MEGA code. Panel (a) shows the poloidal velocity frequency spectrum including all the frequency components, and panel (b) shows the poloidal velocity time evolution including only $50 \mathrm{kHz}$ (red) and $100 \mathrm{kHz}$ (green). Panels (c) and (d) are similar to (a) and (b), but the MHD equations are linearized from $t=1.253 \mathrm{~ms}$.

are consistent with the experiments, as shown in Fig. 5 of Ref. [14].

The mode profiles of poloidal velocity $v_{\theta}$ and bulk pressure perturbation $\delta P_{\text {bulk }}$ are plotted in 3-dimensional figures, as shown in Fig. 3. The five slices in each panel represent five poloidal cross-sections, and their toroidal positions are from $\phi=0$ to $\phi=0.4 \pi$ with toroidal interval of $0.1 \pi$. For $v_{\theta}$, the red color represents positive value. In other words, the red color represents the counter-clockwise rotation in poloidal direction, while the blue color represents the clockwise rotation. For $\delta P_{b u l k}$, the red color represents positive perturbation while the blue color represents negative perturbation. Figure 3 shows that the dominant components of $v_{\theta}$ and $\delta P_{b u l k}$ are $m / n=0 / 0$ and $1 / 0$, respectively. Also, further anal- 

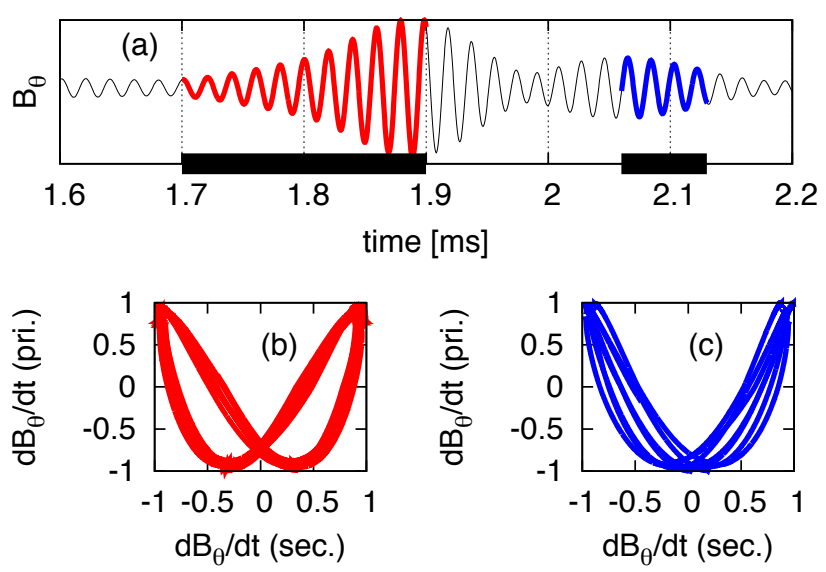

FIG. 2. Lissajous curves between $d B_{\theta} / d t$ values associated with the primary and the secondary modes. Panel (a) represents the time evolution of the secondary mode, while panels (b) and (c) correspond to the Lissajous curves in the growth phase (red) and the decay phase (blue), respectively.

yses show that both the primary mode and the secondary mode are global. The mode number and the mode structure are consistent with the experiment, as shown in Fig. 4 of Ref. [14].

The poloidal velocity $v_{\theta}$ is a combination of $m / n=0 / 0$ (strong), 1/0 (medium), and 2/10 (weak) components. The $m / n=2 / 10$ component exists due to the LHD configuration, because in LHD there are ten pitches in the toroidal direction and there are two high field regions and two low field regions in the poloidal direction. This is the first simulation of EGAM in the 3-dimensional LHD configuration. The mode number is different from the tokamak case where the $v_{\theta}$ oscillation is a combination of $m / n=0 / 0$ and $1 / 0$ components.

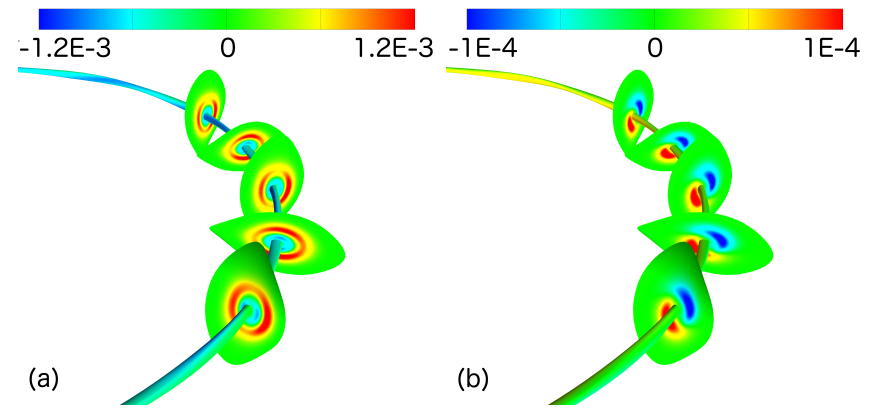

FIG. 3. The mode profiles of (a) $v_{\theta}$ and (b) $\delta P_{b u l k}$ in the 3-dimensional form.

The secondary mode is identified as an EGAM in the present work for three reasons. First, the poloidal mode number is $m=0$ for poloidal velocity and $m=1$ for pressure perturbation. This is the feature of the EGAM and the conventional GAM. Second, the mode frequency is almost the same as the conventional GAM frequency. According to the theoretical prediction, under the present simulation conditions, the conventional GAM frequency should be $50.1 \mathrm{kHz}$. The simulated frequency of the secondary mode is $51 \pm 1.5 \mathrm{kHz}$. And third, the secondary mode is global. The EGAM is global while the conventional GAM is local. This is one of the differences between the EGAM and the conventional $\operatorname{GAM}[7,15,18,20]$. The global structure is caused by the large energetic particle orbit width $[8,9,15,18,20]$. The coupling strength between energetic particles and conventional GAM changes by bulk plasma temperature and GAM continuum, and thus, the mode structure can also be affected by bulk plasma[4, 15, 19]. Based on the three properties discussed above, we conclude that the simulated secondary mode is an EGAM. A question may arise regarding why the primary EGAM and the secondary EGAM have different frequencies. In order to clarify the reason, the bulk plasma pressure perturbation $\delta P_{b u l k}$ and the energetic particle pressure perturbation $\delta P_{h \|}$ are analyzed, as shown in Fig. 4. The most dominant component of $\delta P_{b u l k}$ and $\delta P_{h \|}$ is $m / n=1 / 0$ sine. For simplicity, only this dominant component $1 / 0$ sine is shown in Fig. 4. For the primary mode, the phase of $\delta P_{b u l k}$ and $\delta P_{h \|}$ are the same, and they enhance each other. The primary mode is driven by both $\delta P_{b u l k}$ and $\delta P_{h \|}$. For the secondary mode, the phase difference between $\delta P_{b u l k}$ and $\delta P_{h \|}$ is $\pi$. In other words, they are in anti-phase, and they cancel each other out. Thus, the frequency of the secondary mode is much lower than the primary mode. The phase of $\delta P_{h \perp}$ is the same as $\delta P_{h \|}$, but the absolute value of $\delta P_{h \perp}$ is much smaller. Thus $\delta P_{h \perp}$ is not shown in the figure.

In Ref. [22], the authors claimed that both the fluid nonlinearity and the kinetic nonlinearity are important for the secondary mode excitation. In order to clarify the importance of the fluid nonlinearity, a special linearized MHD model is applied in the present letter. The linear MHD equations are the same as those in Ref. [23]. In the present work, simulations are performed in two stages. In the first stage, the nonlinear code is run until time $t=1.253 \mathrm{~ms}$ when the EGAM is completely saturated but the secondary mode has not yet been excited. Then, in the second stage, both the linear and the nonlinear MHD codes are run separately from the end of the run of the first stage. In the second stage, the secondary mode appears in both runs. In other words, the secondary mode can be excited even if the MHD equations are linearized. In the linearized MHD run, the appearance of the secondary mode is delayed, but the amplitude is almost the same. This result is different from that in Ref. [22]. In the present work, the excitation of the secondary mode is only caused by the kinetic nonlinearity, while the fluid nonlinearity hardly affects the amplitude of the secondary mode.

In order to determine the reason why the frequency 

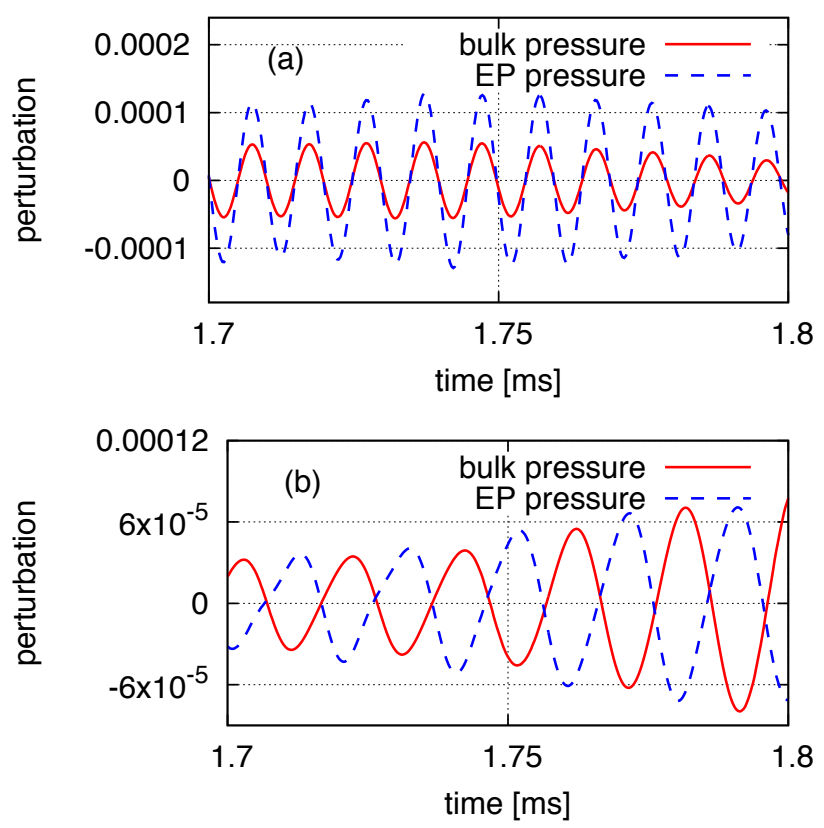

FIG. 4. The $\delta P_{b u l k}$ oscillation and the $\delta P_{h \|}$ oscillation of (a) primary mode and (b) secondary mode.

relation between primary mode and secondary mode is exactly double that which is shown in Fig. 2, and also to further confirm the role of the kinetic nonlinearity, the energy transfer versus transit frequencies of resonant particles are plotted in Fig. 5. Three lines correspond to different phases of the secondary mode: before the excitation, during the growth, and at the time of the beginning of decay at maximum amplitude. The poloidal transit frequency is defined by $f_{t r}=$ $\sqrt{(1-\Lambda)\left(2 E / m_{E P}\right)} /\left(2 \pi q R_{0}\right)$, where $m_{E P}$ is the energetic particle mass. The negative $d E / d t$ indicates that the energetic particles lose energy, and the energy is transferred to the mode, and thus the mode is destabilized. We see in the figure that the energy transfer is strong when the secondary mode is growing at $t=1.75 \mathrm{~ms}$. This indicates that the energy transfer from the particles with $f_{t r} \approx 100 \mathrm{kHz}$ excited the secondary mode with $f \approx 50 \mathrm{kHz}$. This interaction may be the nonlinear resonance. For the linear resonance, the phase of the wave is the same when the particle passes each time around the poloidal angle[27], while the phase is the same when the particle passes $K(K>1)$ times around the poloidal angle for the nonlinear resonance. The nonlinear resonance is negligible for the linear stability analysis, but may transfer substantial energy for finite-amplitude waves. Nonlinear resonance is also called higher-order resonance or fractional resonance, and is commonly known[28]. Fractional resonances were demonstrated experimentally and computationally for EGAMs[8, 21]. For the excitation of the secondary mode shown in Fig. 1, the particles with $f_{t r} \approx 100 \mathrm{kHz}$ satisfy the linear resonance condition for the primary mode with $f \approx 100 \mathrm{kHz}$ and the nonlinear resonance condition for the secondary mode with $f \approx 50 \mathrm{kHz}$ and $K=2$. These resonant particles may transfer energy from the primary mode to the secondary mode, which can be inferred from the decay of the primary mode when the secondary mode is excited.

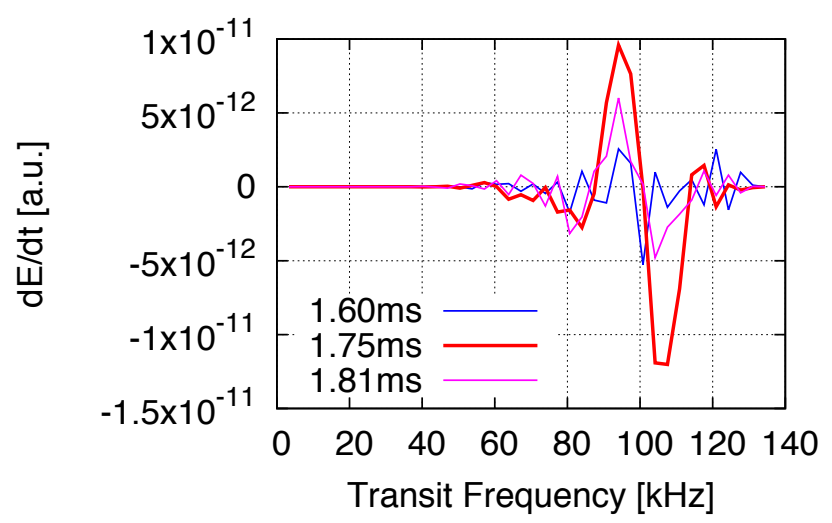

FIG. 5. The energy transfer versus transit frequencies of resonant particles at $1.60 \mathrm{~ms}, 1.75 \mathrm{~ms}$, and $1.81 \mathrm{~ms}$.

In summary, three conclusions are presented in this work. First, the simulation of EGAM in the realistic 3dimensional equilibrium is obtained for the first time, and the results are very similar to the experimental observation. It is found that the poloidal velocity oscillation is a combination of $m / n=0 / 0$ (strong), $1 / 0$ (medium), and $2 / 10$ (weak) components. This is caused by the LHD configuration, and is different from the tokamak case. Second, the chirping EGAM and the associated half-frequency secondary mode are reproduced with the 3-dimensional model and realistic parameters for the first time. The results are good validations of the simulation. It is found that the phase difference between $\delta P_{b u l k}$ and $\delta P_{h \|}$ is $\pi$ for the secondary mode. The $\delta P_{b u l k}$ and $\delta P_{h \|}$ are in anti-phase, and they cancel each other out. Thus, the frequency of the secondary mode is much lower than the primary mode. And third, it is found that the fluid nonlinearity does not affect the excitation of the secondary mode, and the secondary mode is excited by the energetic particles that can resonate with both the primary and the secondary modes. This conclusion is confirmed by the linearized MHD run and by the analysis of the energy transfer from energetic particles to the mode. Our conclusion is different from that of Ref.[22]. However, we would like to emphasize that our simulations are based on the fundamental physics equations with the realistic condition.

We have found that the secondary mode is excited by energetic particles that satisfy both the linear and nonlinear resonance conditions for the primary and the secondary modes, respectively. The overlap of linear and nonlinear resonances is brought about by the sponta- 
neous frequency chirping of the primary mode, and leads to the emergence of stochasticity and the sudden excitation of the secondary mode. The overlap of linear and nonlinear resonances can be ubiquitous in fusion plasmas and important for plasma confinement and energy channeling.

Numerical computations were performed on the Plasma Simulator of NIFS with the support and under the auspices of the NIFS Collaboration Research program (NIFS17KNST111), the Helios of the International Fusion Energy Center, and the K Computer of the RIKEN Advanced Institute for Computational Science (Project ID: hp170260). This work was partly supported by the JSPS-NRF-NSFC A3 Foresight Program in the field of Plasma Physics (NRF: No. 2012K2A2A6000443, NSFC: No.11261140328). The authors thank Prof. M. Osakabe, Prof. H. Sugama, Prof. Y. Idomura, Prof. M. Yokoyama, Dr. M. Lesur, Prof. G. Fu, and Prof. W. Chen for fruitful discussions.

*wanghao@nifs.ac.jp

[1] N. Winsor, J. L. Johnson and J. M. Dawson, Phys. Fluids 11, 2448 (1968).

[2] P. H. Diamond, S.-I. Itoh, K. Itoh, and T. S. Hahm, Plasma Phys. Controlled Fusion 47, R35 (2005).

[3] H. Sugama and T.-H. Watanabe, Phys. Plasmas 13, 012501 (2006).

[4] F. Zonca and L. Chen, Europhysics Lett. 83, 35001 (2008).

[5] C. Boswell, H. Berk, D. Borba, T. Johnson, S. Pinches, and S. Sharapov, Physics Letters A 358, 154 (2006).

[6] H. Berk, C. Boswell, D. Borba, A. Figueiredo, T. Johnson, M. Nave, S. Pinches, S. Sharapov, and JET EFDA contributors, Nuclear Fusion 46, S888 (2006).

[7] R. Nazikian, G. Y. Fu, M. E. Austin, H. L. Berk, R. V. Budny, N. N. Gorelenkov, W. W. Heidbrink, C. T. Holcomb, G. J. Kramer, G. R. McKee, M. A. Makowski, W. M. Solomon, M. Shafer, E. J. Strait, and M. A. Van Zeeland, Phys. Rev. Lett. 101, 185001 (2008).

[8] G. J. Kramer, L. Chen, R. K. Fisher, W. W. Heidbrink, R. Nazikian, D. C. Pace, and M. A. Van Zeeland, Phys. Rev. Lett. 109, 035003 (2012).

[9] R.K. Fisher, D. C. Pace, G. J. Kramer, M. A. Van Zeeland, R. Nazikian, W. W. Heidbrink, and M. GarciaMunoz, Nucl. Fusion 52, 123015 (2012).

[10] K. Toi, F. Watanabe, T. Tokuzawa, K. Ida, S. Morita, T. Ido, A. Shimizu, M. Isobe, K. Ogawa, D. A. Spong, Y. Todo, T. Watari, S. Ohdachi, S. Sakakibara, S. Yamamoto, S. Inagaki, K. Narihara, M. Osakabe, K. Nagaoka, Y. Narushima, K. Y. Watanabe, H. Funaba, M. Goto, K. Ikeda, T. Ito, O. Kaneko, S. Kubo, S. Murakami, T. Minami, J. Miyazawa, Y. Nagayama, M. Nishiura, Y. Oka, R. Sakamoto, T. Shimozuma, Y. Takeiri, K. Tanaka, K. Tsumori, I. Yamada, M. Yosh- inuma, K. Kawahata, and A. Komori, Phys. Rev. Lett. 105, 145003 (2010).

[11] T. Ido, A. Shimizu, M. Nishiura, S. Nakamura, S. Kato, H. Nakano, Y. Yoshimura, K. Toi, K. Ida, M. Yoshinuma, S. Satake, F. Watanabe, S. Morita, M. Goto, K. Itoh, S. Kubo, T. Shi- mozuma, H. Igami, H. Takahashi, I. Yamada, K. Narihara, and the LHD Experiment Group, Nuclear Fusion 51, 073046 (2011).

[12] M. Osakabe, T. Ido, K. Ogawa, A. Shimizu, M. Yokoyama, R. Seki, C. Suzuki, M. Isobe, K. Toi, D. A. Spong, K. Nagaoka, Y. Takeiri, H. Igami, T. Seki, K. Nagasaki, and LHD Experiment Group, "Indication of bulk-ion heating by energetic particle driven Geodesic Acoustic Modes on LHD", presented at The 25th IAEA Fusion Energy Conference Proceedings, St. Petersburg, 2014.

[13] T. Ido, M. Osakabe, A. Shimizu, T. Watari, M. Nishiura, K. Toi, K. Ogawa, K. Itoh, I. Ya- mada, R. Yasuhara, Y. Yoshimura, S. Kato, and the LHD Experiment Group, Nuclear Fusion 55, 083024 (2015).

[14] T. Ido, K. Itoh, M. Osakabe, M. Lesur, A. Shimizu, K. Ogawa, K. Toi, M. Nishiura, S. Kato, M. Sasaki, K. Ida, S. Inagaki, S.-I. Itoh, and the LHD Experiment Group, Phys. Rev. Lett. 116, 015002 (2016).

[15] L. Horvath, G. Papp, Ph. Lauber, G. Por, A. Gude, V. Igochine, B. Geiger, M. Maraschek, L. Guimarais, V. Nikolaeva, G.I. Pokol, and the ASDEX Upgrade Team, Nucl. Fusion 56, 112003 (2016).

[16] D. Zarzoso, Y. Sarazin, X. Garbet, R. Dumont, A. Strugarek, J. Abiteboul, T. Cartier-Michaud, G. DifPradalier, Ph. Ghendrih, V. Grandgirard, G. Latu, C. Passeron, and O. Thomine, Phys. Rev. Lett. 110, 125002 (2013).

[17] M. Sasaki, K. Itoh, K. Hallatschek, N. Kasuya, M. Lesur, Y. Kosuga, and S.-I. Itoh, Scientific Reports 7, 16767 (2017).

[18] G. Y. Fu, Phys. Rev. Lett. 101, 185002 (2008).

[19] Z. Qiu, F. Zonca, and L. Chen, Plasma Sci. Technol. 13, 257 (2011).

[20] H. Wang and Y. Todo, Phys. Plasmas 20, 012506 (2013).

[21] H. Wang, Y. Todo, T. Ido, and M. Osakabe, Phys. Plasmas 22, 092507 (2015).

[22] M. Lesur, K. Itoh, T. Ido, M. Osakabe, K. Ogawa, A. Shimizu, M. Sasaki, K. Ida, S. Inagaki, S.-I. Itoh, and the LHD Experiment Group, Phys. Rev. Lett. 116, 015003 (2016).

[23] Y. Todo, H. Berk, and B. Breizman, Nucl. Fusion 50, 084016 (2010).

[24] H. Wang, Y. Todo, and C. C. Kim, Phys. Rev. Lett. 110, 155006 (2013).

[25] Y. Todo, R. Seki, D. A. Spong, H. Wang, Y. Suzuki, S. Yamamoto, N. Nakajima, and M. Osakabe, Phys. Plasmas 24, 081203 (2017).

[26] Y. Suzuki, N. Nakajima, K. Watanabe, Y. Nakamura, and T. Hayashi, Nucl. Fusion 46, L19 (2006).

[27] Y. Todo and T. Sato, Phys. Plasmas 5, 1321 (1998).

[28] G. J. Lewak and C. S. Chen, J. Plasma Phys. 3, 481 (1969). 